Article

\title{
Resveratrol Trimers from Seed Cake of Paeonia rockii
}

\section{Pu Liu, Yiran Wang, Jiayu Gao, Zongyuan Lu, Weiping Yin * and Ruixue Deng *}

Chemical Engineering \& Pharmaceutical College, Henan University of Science and Technology, Research Center on Wild Natural Resources of FUNIU Mountain, Luoyang 471023, China; E-Mails: liuputju@163.com (P.L.); gjiayu@126.com (Y.W.); cruise1204@163.com (J.G.); zuyuang@126.com (Z.L.)

* Authors to whom correspondence should be addressed; E-Mails: ywpq@163.com (W.Y.); dengliu20022002@aliyun.com (R.D.); Tel.: +86-379-6423-1914 (R.D.); Fax: +86-379-6423-2193 (R.D.).

External Editor: Arthur S. Polans

Received: 11 October 2014; in revised form: 7 November 2014 / Accepted: 18 November 2014 / Published: 26 November 2014

\begin{abstract}
In the course of screening natural products for antibacterial activities, a total acetone extract of the seed cake of Paeonia rockii showed significant effects against bacterial strains. Bioactivity-guided fractionation of the EtOAc-soluble fraction of the total acetone extract resulted in the isolation and identification of five resveratrol trimers, including rockiiol C (1), gnetin H (2), suffruticosol A (3), suffruticosol B (4) and suffruticosol C (5). The relative configuration of these compounds was elucidated mainly by comprehensive $1 \mathrm{D}$ and 2D-NMR experiments. Compound 1 was a new compound. All isolated compounds exhibited strong antibacterial activities against Gram-positive bacteria.
\end{abstract}

Keywords: seed cake; Paeonia rockii; resveratrol trimers; antibacterial activity

\section{Introduction}

Paeonia rockii subsp. rockii cv "spp." (Paeoniaceae) is a shrub widely distributed in China. The seeds oil (peony seed oil) from seeds of Paeonia ostii or Paeonia rockii has been authorized as a new resource food by the Ministry of Health of the People's Republic of China (Bulletin of the Ministry of Health of the People's Republic of China announced, 2011, No. 9). The seed cake is the major by-product in the preparation procedure of crushing cooking oil from the seeds of Paeonia rockii. Though no reports were 
found regarding the chemical constituents of the seed cake of $P$. rockii, studies on the seeds of other Paeonia genera have been performed elsewhere [1-7].

In the course of screening natural products for antibacterial activities, it was found that the acetone extract from the seed cake of $P$. rockii had significant inhibitory effects on bacterial strains. Further bioactivity-guided fractionation led to the isolation and identification of one new resveratrol trimer, rockiiol C (1), together with the four known ones. Their structures are depicted in Figure 1. Herein, we mainly describe the isolation and structural elucidation of the new resveratrol trimer on the basis of various 2D-NMR techniques, including HSQC, HMBC, ${ }^{1} \mathrm{H}-{ }^{1} \mathrm{H}$ COSY and NOESY experiments.

Figure 1. Structures of Compounds 1-5.
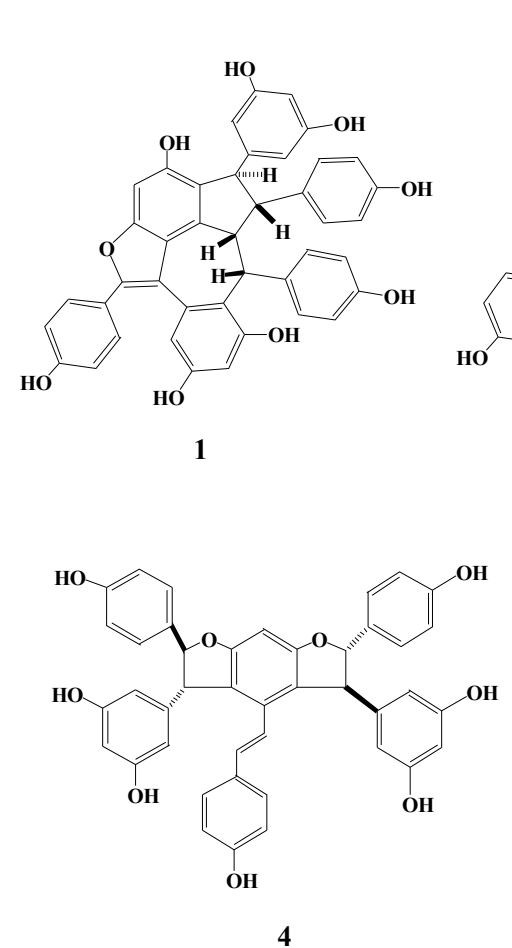

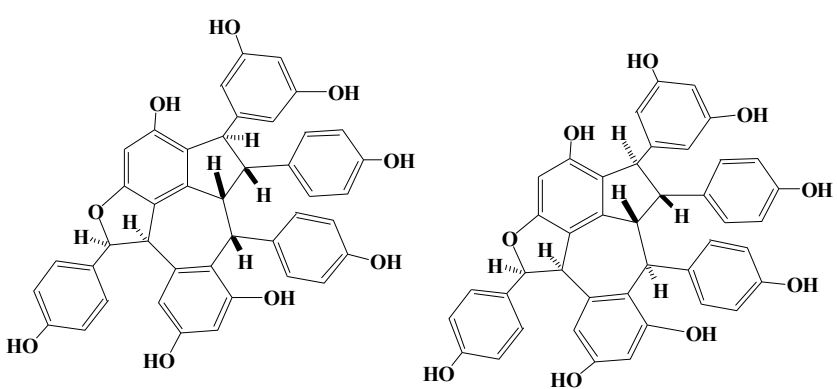

2
3

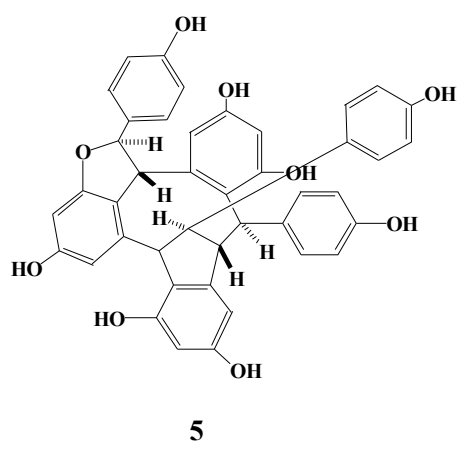

\section{Results and Discussion}

\subsection{Purification and Characterization}

The EtOAc-soluble fraction of the acetone extract from the seed cake of $P$. rockii was separated by silica-gel, gel permeation chromatography and pre-HPLC (ODS-A) to give one new resveratrol trimer, rockiiol C (1), and four known compounds (2-5) (Figure 1).

\subsection{Structural Elucidation of Compound $\mathbf{1}$}

Compound 1, was obtained as a brownish-white amorphous powder and showed a positive reaction with Gibbs reagent. The HRESI-MS data (pseudomolecular ion $[\mathrm{M}+\mathrm{H}]^{+}$at $\mathrm{m} / \mathrm{z} 679.1965$ (calcd. 679.1970)) together with the ${ }^{13} \mathrm{C}-\mathrm{NMR}$ data (42 carbons) indicated the molecular formula $\mathrm{C}_{42} \mathrm{H}_{30} \mathrm{O}_{9}$, which suggested that $\mathbf{1}$ was a resveratrol trimer. The IR spectrum of Compound $\mathbf{1}$ revealed the presence of hydroxy $\left(3336 \mathrm{~cm}^{-1}\right)$ and aromatic rings $\left(1632,1515\right.$ and $\left.1450 \mathrm{~cm}^{-1}\right)$. The UV spectrum displayed an absorption maximum at $289 \mathrm{~nm}$, which was consistent with one or more non-conjugated phenyl rings. 
The ${ }^{1} \mathrm{H}$ - and ${ }^{13} \mathrm{C}-\mathrm{NMR}$ spectrum data (Table 1) exhibited six sets of AX-type hydrogen $(\delta 7.41(\mathrm{~d}$, $J=8.8 \mathrm{~Hz}), \delta 6.75(\mathrm{~d}, J=8.8 \mathrm{~Hz}), \delta 6.68(\mathrm{~d}, J=8.5 \mathrm{~Hz}), \delta 6.26(\mathrm{~d}, J=8.5 \mathrm{~Hz}), \delta 6.17(\mathrm{~d}, J=8.7 \mathrm{~Hz})$, $\delta 6.02(\mathrm{~d}, J=8.7 \mathrm{~Hz}))$ two sets of meta-coupled aromatic hydrogen $(\delta 6.54(\mathrm{~d}, J=2.5 \mathrm{~Hz}), \delta 6.20(\mathrm{~d}$, $J=2.5 \mathrm{~Hz}), \delta 6.08(\mathrm{~d}, J=2.1 \mathrm{~Hz}), \delta 6.04(\mathrm{~d}, J=2.1 \mathrm{~Hz}))$ and singlet aromatic hydrogen $(\delta 6.82(\mathrm{~s}))$. Compound 1 showed four aliphatic signals at $\delta 5.25(\mathrm{~d}, J=3.3 \mathrm{~Hz}), \delta 4.35(\mathrm{dd}, J=3.3,9.1 \mathrm{~Hz}), \delta 3.78$ $(\mathrm{dd}, J=1.5,9.1 \mathrm{~Hz})$ and $\delta 4.62(\mathrm{~d}, J=1.5 \mathrm{~Hz})$, whereas Compounds 2 and $\mathbf{3}$, exhibited six aliphatic hydrogens. Complete assignment of all protons and carbons was confirmed by ${ }^{13} \mathrm{C}-{ }^{1} \mathrm{H}$ COSY. All one- and two-dimensional NMR spectroscopic analyses data indicated that Compound $\mathbf{1}$ is a dehydro derivative of Compounds $\mathbf{2}$ and $\mathbf{3}$. In comparison, for Compounds $\mathbf{2}$ and $\mathbf{3}$, a similar HMBC correlation pattern was revealed in Compound 1, although some differences arose from the unsaturated benzofuran structure. The spectrum data of Compound 1 were similar to that of melapinol B [8], which suggested that 1 was also an oxidative trimer of resveratrol, and the planar structure was the same as that of melapinol B. The planar structure of 1 was confirmed by the one- and two-dimensional NMR spectroscopic analyses data. The spectrum data of the partial structure (C1-C2 and B1-B2) of Compound 1 were similar to those of melapinol B [8], which indicated that they have the same partial structure.

Table 1. ${ }^{1} \mathrm{H}$ - and ${ }^{13} \mathrm{C}-\mathrm{NMR}$ data of $\mathbf{1}$, together with $\mathrm{HMBC}(\mathrm{H} \rightarrow \mathrm{C})$ correlations. At 400/100 MHz, respectively, in $\mathrm{CD}_{3} \mathrm{OD}$; $\delta$ in ppm, $J$ in $\mathrm{Hz}$.

\begin{tabular}{|c|c|c|c|c|c|c|c|}
\hline No. & ${ }^{1} \mathbf{H} \delta J(\mathrm{~Hz})$ & ${ }^{13} \mathrm{C}$ & HMBC & No. & ${ }^{1} \mathrm{H} \delta J(\mathrm{~Hz})$ & ${ }^{13} \mathrm{C}$ & HMBC \\
\hline 1 & & 135.1 & & $8^{\prime}$ & $4.35 \mathrm{dd}, 3.3,9.1$ & 51.8 & $9^{\prime}, 10^{\prime}, 1^{\prime}$ \\
\hline 2 & $6.68 \mathrm{~d}, 8.5$ & 130.8 & $7,1,4$ & $9^{\prime}$ & & 141.5 & \\
\hline 3 & $6.26 \mathrm{~d}, 8.5$ & 115.0 & 1,4 & $10^{\prime}$ & & 120.6 & \\
\hline 4 & & 156.2 & & $11^{\prime}$ & & 154.6 & \\
\hline 5 & $6.26 \mathrm{~d}, 8.5$ & 115.0 & & $12^{\prime}$ & $6.82 \mathrm{~s}$ & 96.4 & $10^{\prime}, 11^{\prime}, 13^{\prime}, 14^{\prime}$ \\
\hline 6 & $6.68 \mathrm{~d}, 8.5$ & 130.8 & & $13^{\prime}$ & & 153.0 & \\
\hline 7 & $3.78 \mathrm{dd}, 1.5,9.1$ & 61.7 & $2,8,9,7^{\prime}$ & $14^{\prime}$ & & 127.9 & \\
\hline 8 & $4.62 \mathrm{~d}, 1.5$ & 58.1 & $7,9,10,8^{\prime}, 14^{\prime}$ & $1^{\prime \prime}$ & & 124.7 & \\
\hline 9 & & 150.0 & & $2^{\prime \prime}$ & $7.41 \mathrm{~d}, 8.8$ & 130.9 & 4",6",7" \\
\hline 10 & $6.08 \mathrm{~d}, 2.1$ & 106.6 & $8,12,14$ & 3" & $6.75 \mathrm{~d}, 8.8$ & 116.3 & $1 ", 4 ", 5 "$ \\
\hline 11 & & 159.3 & & $4^{\prime \prime}$ & & 159.1 & \\
\hline 12 & $6.04 \mathrm{t}, 2.1$ & 101.3 & 10,11 & $5^{\prime \prime}$ & $6.75 \mathrm{~d}, 8.8$ & 116.3 & 4".2",7" \\
\hline 13 & & 159.3 & & $6^{\prime \prime}$ & $7.41 \mathrm{~d}, 8.8$ & 130.9 & 1",4",3" \\
\hline 14 & $6.08 \mathrm{~d}, 2.1$ & 106.6 & & $7^{\prime \prime}$ & & 152.4 & 2",9" \\
\hline 1 & & 136.4 & & $8^{\prime \prime}$ & & 116.4 & $1^{\prime \prime}, 10^{\prime}, 7^{\prime \prime}$ \\
\hline $2^{\prime}$ & $6.17 \mathrm{~d}, 8.7$ & 131.2 & $7^{\prime}, 6^{\prime}, 4^{\prime}$ & $9 " \prime$ & & 141.2 & \\
\hline $3^{\prime}$ & $6.02 \mathrm{~d}, 8.7$ & 114.6 & $1^{\prime}, 4^{\prime}$ & $10^{\prime \prime}$ & & 126.3 & \\
\hline $4^{\prime}$ & & 154.6 & & $11^{\prime \prime}$ & & 155.7 & \\
\hline $5^{\prime}$ & $6.02 \mathrm{~d}, 8.7$ & 114.6 & $1^{\prime}, 4^{\prime}$ & $12 "$ & $6.20 \mathrm{~d}, 2.5$ & 102.5 & $10 ", 11 ", 14 "$ \\
\hline $6^{\prime}$ & $6.17 \mathrm{~d}, 8.7$ & 131.2 & $7^{\prime}, 2^{\prime}, 4^{\prime}$ & $13^{\prime \prime}$ & & 155.7 & \\
\hline $7^{\prime}$ & $5.25 \mathrm{~d}, 3.3$ & 42.1 & $1^{\prime}, 2^{\prime}, 8^{\prime}, 9^{\prime}, 9 ", 10^{\prime \prime}$ & $14^{\prime \prime}$ & $6.54 \mathrm{~d}, 2.5$ & 110.0 & 8",10",12",13" \\
\hline
\end{tabular}

The NOESY (Figure 2) cross peaks between $\mathrm{H}-7^{\prime} / \mathrm{H}-8^{\prime}$ indicated that the rings, $\mathrm{H}-7^{\prime}$ and $\mathrm{H}-8^{\prime}$, were in the $\beta$-configuration as in melapinol B [8]. The significant NOEs between $\mathrm{H}-8^{\prime} / \mathrm{H}-7$ indicated that the relative configuration of the methine proton at C-7 was $\beta$-configuration. Compound 1 showed NOE 
interactions between $\mathrm{H}-8$ and $\mathrm{H}-10$, between $\mathrm{H}-7$ and $\mathrm{H}-2(6)$ and between $\mathrm{H}-7$ and $\mathrm{H}-8$, which is proof of the trans-configuration between $\mathrm{H}-7$ and $\mathrm{H}-8$. Therefore, the methine proton at $\mathrm{C}-8$ was in the $\alpha$-configuration. The assignment of the relative configuration of 1 was $7 R, 8 R, 7^{\prime} R, 8^{\prime} R$, according to the NOE correlations of Compound 1 and the configuration of melapinol B. Therefore, the structure of 1 was designated as shown in Figure 1.

Figure 2. The key NOESY correlations of Compound 1.

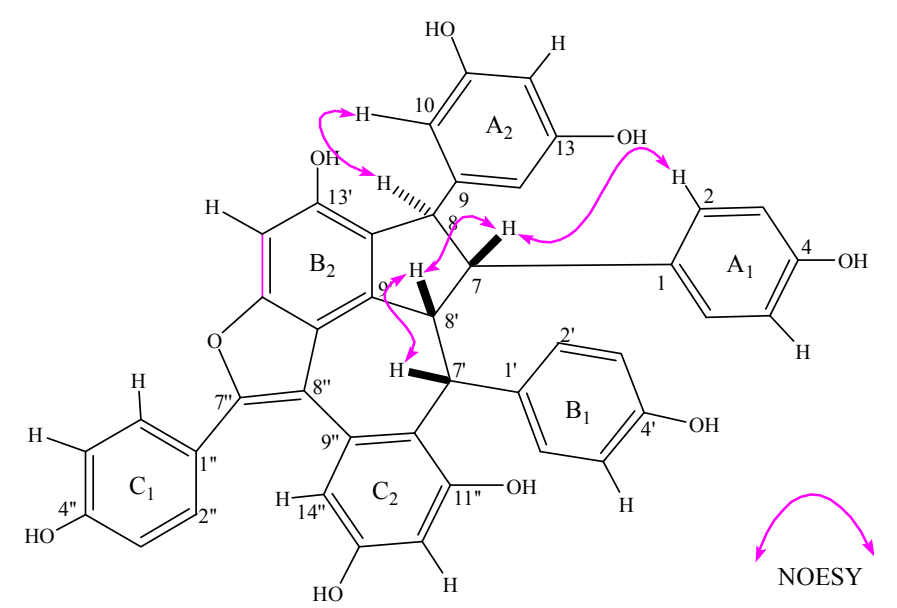

The other known resveratrol oligomers were isolated, and their structures were identified as suffruticosol A (2) [2], suffruticosol B (3) [2], gnetin H (4) [9] and suffruticosol C (5) [2].

\subsection{Antibacterial Activities}

The in vitro anti-bacterial effects of the isolated compounds (1-5) were tested. It was found that all of these compounds exhibited strong antibacterial activities against Gram-positive bacteria. The MIC values against bacterial strains of Compounds $\mathbf{1}-\mathbf{5}$ are listed in Table 2.

Table 2. The antibacterial activities of the compounds (MIC, $\mu \mathrm{g} / \mathrm{mL}$ ).

\begin{tabular}{ccccccc}
\hline Compound & $\begin{array}{c}\text { Staphylococcus } \\
\text { aureus }\end{array}$ & $\begin{array}{c}\text { Pyogenic } \\
\text { streptococcus }\end{array}$ & $\begin{array}{c}\text { Streptococcus } \\
\text { viridans }\end{array}$ & $\begin{array}{c}\text { Staphylococcus } \\
\text { epidermidis }\end{array}$ & $\begin{array}{c}\text { Escherichia } \\
\text { coli }\end{array}$ & $\begin{array}{c}\text { Pseudomonas } \\
\text { aeruginosa }\end{array}$ \\
\hline Penicillin G & 10 & 10 & 10 & 10 & 20 & 10 \\
$\mathbf{1}$ & 20 & 20 & 25 & 20 & 200 & 200 \\
$\mathbf{2}$ & 20 & 20 & 25 & 20 & 200 & 100 \\
$\mathbf{3}$ & 20 & 20 & 25 & 25 & 100 & 200 \\
$\mathbf{4}$ & 25 & 25 & 25 & 20 & 200 & 200 \\
$\mathbf{5}$ & 25 & 30 & 20 & 25 & 100 & 100 \\
\hline
\end{tabular}

\section{Experimental Section}

\subsection{General Experimental Procedures}

Optical rotation was measured with a MC 241 digital polarimeter (Perkin-Elmer, Waltham, MA, USA). IR spectra were recorded on a Perkin-Elmer-577 spectrometer infrared Fourier transform spectrometer (Perkin-Elmer, Waltham, MA, USA). NMR spectra were performed on a Bruker AVANCE 
400 instrument with tetramethylsilane as an internal standard (Rheinstetten, Germany), at $400\left({ }^{1} \mathrm{H}\right)$ and $100 \mathrm{MHz}\left({ }^{13} \mathrm{C}\right)$. HR-ESI-MS was obtained on a Waters LCT-Premier instrument (Milford, MA, USA). HPLC was performed using a Waters 600 with Waters TP pump, UV-2487 detector (Milford, MA, USA) and an YMC-Pack ODS-A column (SH-343-5, Tokyo, Japan). Column chromatography (CC) was performed on silica gel (Qingdao Marine Chemical Co., Ltd, Qingdao, China), Toyopearl HW-40 (TOSOH, Tokyo, Japan). Thin-layer chromatography (TLC) was performed on silica gel GF254 plates (Qingdao Marine Chemical Co., Ltd, Qingdao, China), visualization under UV light and by spraying with $\mathrm{Ce}_{2} \mathrm{SO}_{4}$ or phosphomolybdic acid hydrate, followed by heating.

\subsection{Plant Material}

The seeds of Paeonia rockii were collected at Luoyang Tuqiao flower and seeding CO. LTD, Luoyang city, Henan province, and were verified by Hou Xiao-gai (College of Agricultural, Henan University of Science and Technology) in September, 2012. A voucher specimen has been deposited in the Specimens Hall of Natural resources of Funiu Mountains, Henan University of Science and Technology. The seed cake was obtained in the preparation procedure of crushing cooking oil from the seeds.

\subsection{Extraction and Isolation}

Air-dried seed cakes of $P$. rockii $(7.5 \mathrm{~kg})$ were extracted by acetone at room temperature, and $500 \mathrm{~g}$ of dry crude residues remained after solvent evaporation in vacuo. The residues were dissolved in methanol, and the methanol dissolved fraction (300 g) was then added into the mixtures (1000 g) of silica and diatomite $(1: 1, \mathrm{~W} / \mathrm{W})$. The whole sample was dried at room temperature and then was fractionated successively with EtOAc and methanol to produce 110-g and 150-g dried fractions, respectively. The EtOAc-soluble fraction of the acetone extract was further repeatedly chromatographed on a silica gel column using a gradient solvent system (petroleum ether (PE)-acetone (8:1, 4:1, 2:1, 1:1)) to obtain 10 fractions.

Fraction 5 (14.4 g) was chromatographed on CC to get five subfractions. Subfraction 5.4 (1400 mg) was separated by semi-preparative HPLC (ODS, $\left.\mathrm{MeOH}-\mathrm{H}_{2} \mathrm{O}, 50: 50\right)$ to obtain Compound 2 (23.1 mg).

Fraction 6 (8.6 g) was separated on semi-preparative HPLC (ODS, $\left.\mathrm{MeOH}-\mathrm{H}_{2} \mathrm{O}, 45: 55\right)$ to afford six subfractions. Compound $3\left(12.3 \mathrm{mg}\right.$ ) and Compound 4 were obtained by HPLC (ODS-A; $\mathrm{MeCN}-\mathrm{H}_{2} \mathrm{O}$ 2:8, $3.0 \mathrm{~mL} / \mathrm{min}$ ) from Subfraction $6.1(2,780 \mathrm{mg})$, and Compounds 1 (9.8 $\mathrm{mg})$ and 5 (300.3 $\mathrm{mg})$ were isolated from Subfraction $6.5(780 \mathrm{mg})$.

Rockiiol C (1): Brownish-white amorphous powder. $[\alpha]_{\mathrm{D}}^{25}=-78.2$ (c 0.62, MeOH). UV (MeOH) $\lambda_{\max }$ (loge): 289 (3.57), 228 (3.98). IR $v_{\max }(\mathrm{KBr}) \mathrm{cm}^{-1}: 3,336,1,632,1,515$ and 1,450. The ${ }^{1} \mathrm{H}-\mathrm{NMR}\left(\mathrm{CD}_{3} \mathrm{OD}\right.$, $400 \mathrm{MHz})$ and ${ }^{13} \mathrm{C}-\mathrm{NMR}\left(\mathrm{CD}_{3} \mathrm{OD}, 100 \mathrm{MHz}\right)$ see Table 1. HRESI-MS: $m / z 679.1965[\mathrm{M}+\mathrm{H}]^{+}$(calcd. for 679.1970).

Suffruticosol A (2): Brownish-white amorphous powder, mp 293-296 ${ }^{\circ} \mathrm{C}$, ESI-MS m/z: 679 [M-H] ${ }^{-}$. ${ }^{1} \mathrm{H}-\mathrm{NMR}\left(\mathrm{CD}_{3} \mathrm{OD}, 400 \mathrm{MHz}\right), \delta: 7.10\left(2 \mathrm{H}, \mathrm{d}, J=8.6 \mathrm{~Hz}, \mathrm{H}-2^{\prime}, 6^{\prime}\right), 6.68$ (2H, d, $\left.J=8.6 \mathrm{~Hz}, \mathrm{H}-3^{\prime}, 5^{\prime}\right)$, 6.47 (2H, d, $J=8.6 \mathrm{~Hz}, \mathrm{H}-2 ", 6 "), 6.11$ (2H, d, $\left.J=8.6 \mathrm{~Hz}, \mathrm{H}-3 ", 5^{\prime}\right), 6.95$ (2H, d, $\left.J=8.2 \mathrm{~Hz}, \mathrm{H}-2,6\right)$, $6.36(2 \mathrm{H}, \mathrm{d}, J=8.2 \mathrm{~Hz}, \mathrm{H}-3,5), 6.25$ (1H, d, $J=1.68 \mathrm{~Hz}, \mathrm{H}-14 "), 5.92(1 \mathrm{H}, \mathrm{d}, J=1.68 \mathrm{~Hz}, \mathrm{H}-12 "), 6.19$ 
$\left(1 \mathrm{H}, \mathrm{d}, J=0.56 \mathrm{~Hz}, \mathrm{H}-12^{\prime}\right), 6.05(1 \mathrm{H}, \mathrm{t}, J=2.16 \mathrm{~Hz}, \mathrm{H}-12), 5.97$ (2H, t, $\left.J=2.16 \mathrm{~Hz}, \mathrm{H}-10,14\right), 3.67$ $(1 \mathrm{H}, \mathrm{d}, J=7.72 \mathrm{~Hz}, \mathrm{H}-7), 4.74$ (1H, brs, H-8), $5.42\left(1 \mathrm{H}, \mathrm{d}, J=3.24 \mathrm{~Hz}, \mathrm{H}-7{ }^{\prime}\right), 3.93$ (1H, m, H-8'), 5.68 $(1 \mathrm{H}, \mathrm{d}, J=15.7 \mathrm{~Hz}, \mathrm{H}-7 "), 4.34(1 \mathrm{H}, \mathrm{d}, J=15.7 \mathrm{~Hz}, \mathrm{H}-8 ") .{ }^{13} \mathrm{C}-\mathrm{NMR}\left(\mathrm{CD}_{3} \mathrm{OD}, 100 \mathrm{MHz}\right) \delta: 135.6(\mathrm{C}-1)$, 130.9 (C-2), 115.5 (C-3), 156.5 (C-4), 115.5 (C-5), 130.9 (C-6), 60.7 (C-7), 54.6 (C-8), 148.4 (C-9), 106.8 (C-10), 159.3 (C-11), 101.4 (C-12), 159.3 (C-13), 106.8 (C-14), 133.9 (C-1'), 130.5 (C-2'), 116.3 (C-3'), 159.0 (C-4'), 116.3 (C-5'), 130.5 (C-6'), 39.7 (C-7'), 49.1 (C-8'), 144.7 (C-9'), 117.3 (C-10'), 160.2 (C-11'), 96.2 (C-12'), 155.0 (C-13'), 123.0 (C-14'), 130.9 (C-1"), 130.8 (C-2"), 114.2 (C-3"), 154.5 (C-4"), 114.2 (C-5"), 130.8 (C-6"), 91.5 (C-7"), 48.6 (C-8"), 141.8 (C-9"), 126.9 (C-10"), 155.2 (C-11"), 105.9 (C-12"), 156.7 (C-13"), 101.9 (C-14").

Suffruticosol B (3): Brownish-white amorphous powder, mp 297-301 ${ }^{\circ} \mathrm{C}$, ESI-MS $m / z$ : 679 [M-H] ${ }^{-}$. ${ }^{1} \mathrm{H}-\mathrm{NMR}\left(\mathrm{CD}_{3} \mathrm{OD}, 400 \mathrm{MHz}\right), \delta: 6.95$ (2H, d, $\left.J=8.6 \mathrm{~Hz}, \mathrm{H}-2^{\prime}, 6^{\prime}\right), 6.49$ (2H, d, $\left.J=8.6 \mathrm{~Hz}, \mathrm{H}-3^{\prime}, 5^{\prime}\right)$, 7.57 (2H, d, $J=8.6 \mathrm{~Hz}, \mathrm{H}-2 ", 6 "), 6.93$ (2H, d, $J=8.6 \mathrm{~Hz}, \mathrm{H}-3 ", 5 "), 6.27$ (2H, d, $J=8.4 \mathrm{~Hz}, \mathrm{H}-2,6$ ), $6.29(2 \mathrm{H}, \mathrm{d}, J=8.2 \mathrm{~Hz}, \mathrm{H}-3,5), 5.94(1 \mathrm{H}, \mathrm{d}, J=2.16 \mathrm{~Hz}, \mathrm{H}-14 "), 6.17$ (1H, d, $J=2.16 \mathrm{~Hz}, \mathrm{H}-12 "), 6.19$ $(1 \mathrm{H}, \mathrm{s}, \mathrm{H}-12 '), 6.14(1 \mathrm{H}, \mathrm{t}, J=2.16 \mathrm{~Hz}, \mathrm{H}-12), 6.22(2 \mathrm{H}, \mathrm{t}, J=2.16 \mathrm{~Hz}, \mathrm{H}-10,14), 3.80(1 \mathrm{H}, \mathrm{d}, J=6.0$ Hz, H-7), 4.08 (1H, m, H-8), 4.22 (1H, d, $J=11.72$ Hz, H-7'), 4.10 (1H, brd, $\left.J=11.76, \mathrm{H}^{\prime} 8^{\prime}\right), 5.86(1 \mathrm{H}$, $\mathrm{d}, J=11.84 \mathrm{~Hz}, \mathrm{H}-7 "), 5.08$ (1H, d, $J=11.84 \mathrm{~Hz}, \mathrm{H}-8 ") .{ }^{13} \mathrm{C}-\mathrm{NMR}\left(\mathrm{CD}_{3} \mathrm{OD}, 100 \mathrm{MHz}\right) \delta: 135.5$ (C-1), 129.5 (C-2), 115.2 (C-3), 156.1 (C-4), 115.2 (C-5), 129.5 (C-6), 63.1 (C-7), 56.9 (C-8), 147.5 (C-9), 107.4 (C-10), 159.4 (C-11), 101.5 (C-12), 159.4 (C-13), 107.4 (C-14), 133.8 (C-1'), 133.1 (C-2'), 114.7 (C-3'), 156.1 (C-4'), 114.7 (C-5'), 133.1 (C-6'), 46.5 (C-7'), 47.8 (C-8'), 144.7 (C-9'), 117.3 (C-10'), 160.2 (C-11'), 96.2 (C-12'), 155.0 (C-13'), 123.6 (C-14'), 130.9 (C-1"), 130.6 (C-2"), 116.5 (C-3"), 159.2 (C-4"), 116.5 (C-5"), 130.6 (C-6"), 91.2 (C-7"), 49.0 (C-8"), 142.4 (C-9"), 122.9 (C-10"), 157.2 (C-11"), 105.0 (C-12"), 1,558.4 (C-13"), 103.7 (C-14").

Gnetin H (4): White powders, mp $185-187^{\circ} \mathrm{C}$, ESI-MS $m / z$ : $679[\mathrm{M}-\mathrm{H}]^{-} .{ }^{1} \mathrm{H}-\mathrm{NMR}\left(\mathrm{CD}_{3} \mathrm{OD}, 400 \mathrm{MHz}\right)$, $\delta: 7.19$ (4H, dd, $\left.J=2.0,8.6 \mathrm{~Hz}, \mathrm{H}-2,6,2^{\prime \prime}, 6 "\right), 6.79$ (4H, dd, $\left.J=2.0,8.6 \mathrm{~Hz}, \mathrm{H}-3,5,3 ", 5 "\right), 6.69$ (2H, dd, $\left.J=2.0,8.6 \mathrm{~Hz}, \mathrm{H}-2^{\prime}, 6^{\prime}\right), 6.51$ (2H, dd, $\left.J=2.0,8.6 \mathrm{~Hz}, \mathrm{H}-3^{\prime}, 5^{\prime}\right), 6.43$ (1H, s, H-12'), 6.38 (2H, brs, H-7', 8'), 6.14 (6H, s, H-10, 12, 14, 10", 12", 14"), 5.41 (2H, d, J=5.64 Hz, H-7, 7"), 4.40 (2H, d, J= 5.64 Hz, $\mathrm{H}-8,8$ "). ${ }^{13} \mathrm{C}-\mathrm{NMR}\left(\mathrm{CD}_{3} \mathrm{OD}, 100 \mathrm{MHz}\right) \delta$ : 134.2 (C-1), 128.1 (C-2), 116.4 (C-3), 158.6 (C-4), 116.4 (C-5), 128.1 (C-6), 94.5 (C-7), 59.0 (C-8), 147.6 (C-9), 107.3 (C-10), 160.1 (C-11), 102.1 (C-12), 160.1 (C-13), 107.3 (C-14), 130.6 (C-1'), 128.7 (C-2'), 116.2 (C-3'), 158.3 (C-4'), 116.2 (C-5'), 128.7 (C-6'), 134.6 (C-7'), 122.5 (C-8'), 128.1 (C-9'), 120.3 (C-10'), 163.0 (C-11'), 91.5 (C-12'), 163.0 (C-13'), 120.3 (C-14'), 133.7 (C-1"), 128.1 (C-2"), 116.4 (C-3"), 158.6 (C-4"), 116.4 (C-5"), 128.1 (C-6"), 94.9 (C-7"), 59.0 (C-8"), 147.6 (C-9"), 107.3 (C-10"), 160.1 (C-11"), 102.1 (C-12"), 160.1 (C-13"), 107.3 (C-14").

Suffruticosol C (5). Brownish-white amorphous powder, mp 197-199 ${ }^{\circ} \mathrm{C}$, ESI-MS $m / z: 679[\mathrm{M}-\mathrm{H}]^{-}$. ${ }^{1} \mathrm{H}-\mathrm{NMR}\left(\mathrm{CD}_{3} \mathrm{OD}, 400 \mathrm{MHz}\right), \delta: 7.20\left(2 \mathrm{H}, \mathrm{d}, J=8.4 \mathrm{~Hz}, \mathrm{H}-2^{\prime}, 6^{\prime}\right), 6.78\left(2 \mathrm{H}, \mathrm{d}, J=8.4 \mathrm{~Hz}, \mathrm{H}-3^{\prime}, 5^{\prime}\right)$, 7.08 (2H, d, $J=8.4 \mathrm{~Hz}, \mathrm{H}-2 ", 6 "), 6.78$ (2H, d, $J=8.4 \mathrm{~Hz}, \mathrm{H}-3 ", 5 "), 6.92$ (2H, d, $J=8.4 \mathrm{~Hz}, \mathrm{H}-2,6)$, $6.51(2 \mathrm{H}, \mathrm{d}, J=8.4 \mathrm{~Hz}, \mathrm{H}-3,5), 6.38(1 \mathrm{H}, \mathrm{d}, J=2.24 \mathrm{~Hz}, \mathrm{H}-10 "), 6.23$ (1H, d, $J=2.24 \mathrm{~Hz}, \mathrm{H}-12 "), 5.98$ $(1 \mathrm{H}, \mathrm{s}, \mathrm{H}-12 '), 6.00(1 \mathrm{H}, \mathrm{t}, J=2.2 \mathrm{~Hz}, \mathrm{H}-12), 5.86(2 \mathrm{H}, \mathrm{t}, J=2.2 \mathrm{~Hz}, \mathrm{H}-10,14), 4.28(1 \mathrm{H}, \mathrm{d}$, $\left.J=10.2 \mathrm{~Hz}, \mathrm{H}-8^{\prime}\right), 2.97$ (1H, m, H-7'), 4.14 (1H, brd, $\left.J=11.72, \mathrm{H}-8\right), 6.00$ (1H, d, $\left.J=1.34 \mathrm{~Hz}, \mathrm{H}-7^{\prime \prime}\right)$, $4.23\left(1 \mathrm{H}, \mathrm{d}, J=1.34 \mathrm{~Hz}, \mathrm{H}-8\right.$ "), 5.07 (1H, brs, H-7). ${ }^{13} \mathrm{C}-\mathrm{NMR}\left(\mathrm{CD}_{3} \mathrm{OD}, 100 \mathrm{MHz}\right) \delta: 138.0$ (C-1), 129.8 
(C-2), 115.5 (C-3), 155.8 (C-4), 115.5 (C-5), 129.8 (C-6), 36.8 (C-7), 52.3 (C-8), 144.0 (C-9), 123.1 (C-10), 158.8 (C-11), 101.3 (C-12), 158.8 (C-13), 107.8 (C-14), 133.0 (C-1'), 130.9 (C-2'), 115.9 (C-3'), 156.9 (C-4'), 115.9 (C-5'), 133.0 (C-6'), 67.0 (C-7'), 57.7 (C-8'), 147.2 (C-9'), 107.8 (C-10'), $159.6\left(\mathrm{C}-11^{\prime}\right), 96.2$ (C-12'), 155.0 (C-13'), 1119.5 (C-14'), 134.8 (C-1"), 128.0 (C-2"), 116.2 (C-3"), 158.2 (C-4"), 116.2 (C-5"), 128.0 (C-6"), 86.3 (C-7"), 51.0 (C-8"), 147.6 (C-9"), 103.5 (C-10"), 157.1 (C-11"), 101.3 (C-12"), 158.9 (C-13"), $118.6(\mathrm{C}-14 ")$.

\subsection{Bioassay}

The MIC (minimum inhibitory concentration) of Compounds 1-5 against the bacterial strains Staphylococcus aureus, pyogenic streptococcus, Streptococcus viridans, Staphylococcus epidermidis, Escherichia coli and Pseudomonas aeruginosa, were determined using the standard broth microdilution method [10,11]. Penicillin G was used as the positive control. The test compounds (50.0 mg) were diluted in $\mathrm{Me}_{2} \mathrm{SO}(500 \mu \mathrm{L})$ and mixed with bacterial strains cultured in nutrient broths $(9.5 \mathrm{~mL})$. The initial concentration of test compounds was $5 \mathrm{mg} / \mathrm{mL}$, and concentrations of 10, 20, 30, 40, 50, 60, 70, $80,100,200,300,400$ and $500 \mu \mathrm{g} / \mathrm{mL}$ were obtained by serial dilutions. Antibacterial tests were performed by transferring each test compound into different concentration $(1 \mathrm{~mL})$ into a new test tube containing only bacterial culture $(1 \mathrm{~mL})$. Observations were made after $24 \mathrm{~h}$ to determine the possible bacterial growth in the respective culture broths. The optical density of treated cells reflects their viability and provides sufficient information pertaining to the mode of action of the tested metabolites.

\section{Conclusions}

In summary, one new compound, $\mathbf{1}$, and four known compounds (2-5) were characterized from the seed cake of Paeonia rockii. These compounds could be classified into resveratrol trimers. Additionally, the basic unit of these compounds was resveratrol. The results revealed that these compounds displayed varying degrees of antibacterial activity. The isolated compounds were all oligostilbenes. Oligostilbenes are a class of plant polyphenols and have attracted intense interest for their intricate structures and diverse biological activities. These compounds and their derivatives are of significant interest for drug research and development, because of their potential in therapeutic or preventive applications, such as anti-bacterial agents, antioxidant and antitumor drugs, and so on.

\section{Supplementary Materials}

Supplementary materials can be accessed at: http://www.mdpi.com/1420-3049/19/12/19549/s1.

\section{Acknowledgments}

This work was supported the National Natural Science Foundation of China (No. U1404831) and the funds from Henan University of Science and Technology (No. ZCX005, CXJJ-Z024). We thank the National Natural Science foundation of China for financial support of this research. 


\section{Author Contributions}

$\mathrm{Pu}$ Liu, Ruixue Deng and Weiping Yin conceptualized the project, and Ruixue Deng headed the project. Pu Liu, Yiran Wang, Jiayu Gao, Zongyuan Lu and Ruixue Deng performed the experiments. $\mathrm{Pu}$ Liu and Ruixue Deng were involved in writing the manuscript.

\section{Conflicts of Interest}

The authors declare no conflict of interest.

\section{References}

1. Qi, J.C.; Zhou, H.M.; Ma, J.Q.; Li, P. Analysis of the chemical constituents in Peony seed oil by GC-MS. Cereals Oils 2005, 19, 22-23.

2. Sarker, S.D.; Whiting, P.; Dinan, L.; Sil, V.; Rees, H.H. Identification and ecdysteroid antagonist activity of three resveratrol trimers (Suffruticosols A, B and C) from Paeonia suffruticosa. Tetrahedron 1999, 55, 513-524.

3. He, C.N.; Xiao, W.; Li, M.; Peng, Y.; Xu, J.L.; Gu, J.; Xiao, P.G. Chemical Constituents from Seeds of Paeonia suffruticosa. China J. Chin. Mater. Med. 2010, 35, 1428-1431.

4. He, C.N.; Zhang, Y.C.; Peng, Y.; Yang, J.S.; Xiao, P.G. Monoterpene glycosides from the seeds of Paeonia suffruticosa protect HEK 293 cells from irradiation-induced DNA damage. Phytochem. Lett. 2012, 5, 128-133.

5. Yi, J.P.; Zhu, W.X.; Ma, H.L.; Wang, Y.F. Studies on Chemical Constituents from Seeds of Paeonia suffruticosa Andr. Nat. Prod. Res. Dev. 2009, 20, 604-607.

6. Wu, S.H.; Wu, D.G.; Chen, Y.W. Chemical Constituents and Bioactivities of Plants from the Genus Paeonia. Chem. Biodivers. 2010, 7, 90-104.

7. He, C.N.; Peng, Y.; Zhang, Y.C.; Xu, L.J.; Gu, J.; Xiao, P.G. Phytochemical and Biological Studies of Paeoniaceae. Chem. Biodivers. 2010, 7, 805-838.

8. Yoshio, H.; Ryuichiro, K.; Kokki, S. Compounds inhibitory to rat liver $5 \alpha$-reductase from tropical commercial wood species: Resveratrol trimers from melapi (Shore sp.) heartwood. J. Wood Sci. 2001, 47, 308-312.

9. Kim, H.J.; Chang, E.J.; Bae, S.J.; Shim, S.M.; Park, H.D.; Rhee, C.H.; Park, J.H.; Choi, S.W. Cytotoxic and AnUmutagenic Stilbenes from Seeds of Paeonia lactiflora. Arch. Pharm. Res. 2002, 25, 293-299.

10. Pavel, N.; Kloucek, P.; Rondevaldova, J.; Havlik, J.; Kourimska, L.; Kokoska, L. Thymoquinone vapor significantly affects the results of Staphylococcus aureus sensitivity tests using the standard broth microdilution method. Fitoterapia 2014, 94, 102-107.

11. Kajal, C.; Lipton, A.P.; Raj, R.P.; Vijayan, K.K. Antibacterial labdane diterpenoids of Ulva fasciata Delile from southwestern coast of the Indian Peninsula. Food Chem. 2010, 119, 1399-1408.

Sample Availability: Samples of the compounds 2-5 are available from the authors.

(C) 2014 by the authors; licensee MDPI, Basel, Switzerland. This article is an open access article distributed under the terms and conditions of the Creative Commons Attribution license (http://creativecommons.org/licenses/by/4.0/). 